\title{
Resultados de la unidad de oncología del Departamento de Cirugía de un hospital universitario (2004-2010)*
}

\author{
E.U. SOLANGE CORTÉS L. ${ }^{1}$, Drs. ATTILA CSENDES J. ${ }^{1}$, JULIO YARMUCH G. ${ }^{1}$ \\ 1 Departamento de Cirugía Hospital Clínico de la Universidad de Chile. \\ Santiago, Chile.
}

\section{Results of the oncology Unit, Department of Surgery of a university hospital}

\section{Introducción}

Los tumores malignos en Chile constituyen la segunda causa ${ }^{1}$ de muerte en la población chilena. De 90.168 fallecidos el año 2008, un 24,2\%, es decir, 21.820 sujetos fallecieron por cáncer. Con el objeto de centralizar toda la información referente al tratamiento quirúrgico de los diferentes tipos de cáncer que se atienden en el Departamento de Cirugía del Hospital Clínico de la Universidad de Chile, se creó en 2004 una unidad a cargo de una enfermera universitaria dedicada a tiempo completo a esta función. El presente estudio muestra los resultados de estos últimos 7 años.

\section{Método}

El presente reporte es un estudio prospectivo que comenzó en mayo de 2004, cuando se creó la Unidad de Oncología a cargo de una enfermera universitaria (S.C.) especializada en cirugía de adultos. Antes de crear esta unidad, concurrió durante un mes a la unidad de seguimiento de cáncer de colon y recto de la Pontificia Universidad Católica. A su regreso se confeccionó una planilla Excel, incorporando múltiples datos de cada cáncer particular, te- niendo una sección general de tipo epidemiológico y una sección particular, con el estudio preoperatorio, la operación efectuada, los cirujanos, la evolución postoperatoria y las complicaciones particulares de cada tumor específico, los completos hallazgos anatomopatológicos, la mortalidad y la evolución alejada con seguimiento actualizado cada 6 meses, tanto del control clínico como de registro civil en caso de defunción. Cada paciente con tumor maligno que ingresa al Departamento es registrado de inmediato en esta unidad, registrando todos los parámetros hasta el momento del alta en forma prospectiva, lo que permite tener un completo estudio en cada paciente. La enfermera encargada de esta unidad recolecta personalmente los informes de anatomía patológica, colocando uno en la ficha y guardando otra copia en esta Unidad.

\section{Resultados}

La Tabla 1 muestra las cifras globales registradas en forma prospectiva en esta Unidad desde mayo de 2004 hasta diciembre de 2010. Hubo un total de 3.015 pacientes atendidos, lo que da un promedio de 37 pacientes con tumores malignos atendidos por mes y cerca de 458 pacientes cada año. El 97\%

\footnotetext{
*Recibido el 14 de marzo de 2011 y aceptado para publicación el 6 de junio de 2011.

Correspondencia: Dr. Attila Csendes J.

Santos Dumont 999, Santiago, Chile. acsendes@redclinicauchile.cl
} 
RESULTADOS DE LA UNIDAD DE ONCOLOGÍA DEL DEPARTAMENTO DE CIRUGÍA DE UN HOSPITAL ...

Tabla 1. Estadísticas globales de la Unidad de Oncología 2004-2010

\begin{tabular}{|lrr|}
\hline Operados & 2.910 & \\
\hline No operados & 105 & \\
\hline Total casos & 3.015 & \\
\hline Género (operados) & & \\
Femenino & 1.785 & $61,3 \%$ \\
Masculino & 1.125 & $38,7 \%$ \\
\hline
\end{tabular}

de estos pacientes fueron sometidos a alguna operación y todas las cifras posteriores se refieren solamente a este grupo, no incluyendo los 105 pacientes no operados. Hubo un predominio global del sexo femenino sobre el masculino, en una proporción de 1,6:1. La Tabla 2 describe la localización original de los tumores malignos operados. Se aprecia que el cáncer de tiroides ocupa un lugar muy preponderante, casi un tercio del total de los tumores malignos. En el segundo lugar se aprecia el cáncer de colon, recto y ano, los que en total corresponden al 21\% de los pacientes.

El cáncer gástrico ocupa el tercer lugar. Llama la atención el alto número de pacientes operados del área hepato-bilio pancreática, ocupando un $17,1 \%$ del total de pacientes operados, es decir, 499 pacientes, o 6,3 pacientes por mes. El cáncer de mama es una patología poco frecuente en nuestro servicio, atendiendo cerca de 8 pacientes por año.

La Tabla 3 describe la distribución por edad promedio de cada cáncer en particular y las edades mínimas y máximas para cada tumor. En este último rubro se aprecia la amplia gama de distribución de edad, que va desde los 12 años hasta más de 90 años.

El cáncer de tiroides es el que con mayor frecuencia se desarrolla en personas jóvenes bajo los 50 años. Hay varios tumores con edades promedio entre los 50 y 60 años, siendo el cáncer de colon y el de esófago, los que se presentan en una edad promedio mayor que el resto.

La Tabla 4 muestra la distribución por sexo de los 15 tumores malignos más frecuentes operados entre 2004 y 2010. Hay 3 tumores que, son significativamente más frecuentes en mujeres
Tabla 2. Localización del tumor maligno (2004-2010)

\begin{tabular}{|llc|}
\hline Ubicación tumor & n & \% \\
Tiroides & 904 & 31,1 \\
\hline Colon & 460 & 15,8 \\
\hline Gástrico & 386 & 13,3 \\
\hline Periampular & 172 & 5,9 \\
\hline Vesícula & 168 & 5,8 \\
\hline Recto & 145 & 5,0 \\
\hline Cabeza y cuello & 77 & 2,6 \\
\hline Pulmón & 75 & 2,6 \\
\hline Hígado & 71 & 2,4 \\
\hline Intestino delgado & 69 & 2,4 \\
\hline Partes blandas & 60 & 2,1 \\
\hline Esófago & 59 & 2,0 \\
\hline Metástasis hepáticas & 52 & 1,8 \\
Mama & 48 & 1,6 \\
\hline Melanoma & 43 & 1,5 \\
\hline Piel & 39 & 1,3 \\
Vía biliar & 36 & 1,2 \\
\hline GIST gástrico & 21 & 0,7 \\
\hline Metástasis pulmón & 18 & 0,6 \\
\hline Anal & 7 & 0,2 \\
\hline Total & 2.910 & \\
\hline & & \\
\hline
\end{tabular}

Tabla 3. Distribución por edad de los tumores malignos (2004-2010)

\begin{tabular}{|lccc|}
\hline Ubicación tumor & $\begin{array}{c}\text { Edad prome- } \\
\text { dio (años) }\end{array}$ & Mínimo & Máximo \\
\hline Tiroides & $45,7 \pm 14,2$ & 12 & 85 \\
Colon & $66,3 \pm 13,6$ & 20 & 94 \\
\hline Gástrico & $61,2 \pm 12,9$ & 26 & 83 \\
\hline Periampular & $59,5 \pm 12,7$ & 23 & 83 \\
\hline Vesícula & $61,9 \pm 11,8$ & 25 & 88 \\
\hline Recto & $61,7 \pm 14,8$ & 17 & 93 \\
\hline Cabeza y cuello & $59,7 \pm 14,8$ & 16 & 86 \\
\hline Pulmón & $61,5 \pm 10,9$ & 31 & 86 \\
\hline Hígado & $62,4 \pm 12,8$ & 18 & 87 \\
\hline Intestino delgado & $55,1 \pm 18,7$ & 15 & 89 \\
\hline Partes blandas & $55,0 \pm 17,8$ & 20 & 88 \\
\hline Esófago & $64,0 \pm 9,8$ & 42 & 82 \\
\hline Metástasis hepáticas & $60,4 \pm 12,6$ & 26 & 82 \\
\hline Mama & $58,9 \pm 14,8$ & 29 & 88 \\
\hline Melanoma & $55,6 \pm 18,2$ & 20 & 88 \\
\hline Piel & $62,4 \pm 14,9$ & 33 & 86 \\
\hline Vía biliar & $61,8 \pm 11,9$ & 37 & 83 \\
\hline GIST gástrico & $62,2 \pm 15,1$ & 31 & 93 \\
\hline Metástasis pulmón & $53,0 \pm 14,4$ & 24 & 75 \\
\hline Anal & $57,6 \pm 15,1$ & 23 & 74 \\
\hline
\end{tabular}


Tabla 4. Distribución por género 2004-2010 de los 15 tumores malignos más frecuentes

\begin{tabular}{|lrrrr|}
\hline Ubicación tumor & \multicolumn{2}{c}{ Mujeres } & \multicolumn{2}{c|}{ Hombres } \\
& n & \% & \multicolumn{1}{c|}{ n } & \% \\
Tiroides & 765 & 84,6 & 139 & 15,4 \\
Colon & 249 & 54,1 & 211 & 45,9 \\
Gástrico & 145 & 37,5 & 241 & 62,5 \\
\hline Periampular & 91 & 52,9 & 81 & 47,1 \\
Vesícula & 119 & 52,4 & 49 & 47,6 \\
Recto & 76 & 70,8 & 69 & 29,2 \\
Cabeza y cuello & 33 & 42,8 & 44 & 57,2 \\
\hline Pulmón & 31 & 41,3 & 44 & 59,7 \\
\hline Hígado & 36 & 50,7 & 35 & 49,3 \\
Intestino delgado & 29 & 42,0 & 40 & 58,0 \\
\hline Partes blandas & 32 & 53,3 & 28 & 46,7 \\
\hline Esófago & 27 & 45,8 & 32 & 54,2 \\
\hline Metástasis hepáticas & 24 & 46,1 & 28 & 53,9 \\
\hline Mama & 47 & 98,0 & 1 & 2,0 \\
\hline Melanoma & 23 & 53,5 & 20 & 46,5 \\
\hline
\end{tabular}

Tabla 5. Mortalidad operatoria de tumores malignos resecados (2004-2010)

\begin{tabular}{|lccc|}
\hline Ubicación & n & \multicolumn{2}{c|}{ Mortalidad } \\
& & n & \% \\
\hline Tiroides & 904 & 4 & 0,4 \\
Colon & 460 & 11 & 2,4 \\
\hline Gástrico & 386 & 11 & 2,8 \\
\hline Periampular & 172 & 5 & 2,9 \\
\hline Vesícula & 168 & 4 & 2,4 \\
\hline Recto & 145 & 2 & 1,4 \\
\hline Pulmón & 75 & 4 & 5,3 \\
\hline Hígado & 71 & 3 & 4,2 \\
\hline Intestino delgado & 69 & 3 & 4,3 \\
\hline Partes blandas & 60 & 2 & 3,3 \\
\hline Esófago & 59 & 3 & 5,1 \\
\hline Metástasis hepáticas & 52 & 3 & 5,8 \\
\hline Vía biliar & 36 & 3 & 8,3 \\
\hline Cabeza y cuello, mama, & 253 & 0 & 0 \\
melanoma, piel, GIST, & & & \\
metástasis pulmón, anal & & & 2,0 \\
\hline Global & 2.910 & 58 & \\
\hline
\end{tabular}

que en hombres: tiroides, vesícula y mama. Por otra parte, el cáncer gástrico es el único más frecuente en hombres. El resto de los tumores tiene una distribución similar en ambos sexos. En la Tabla 5 se detalla la mortalidad operatoria, definida hasta los 30 días o durante la hospitalización si esta era mayor a 30 días. La mortalidad global es de un 2,0\%. Hay algunas intervenciones de alta complejidad como la esofaguectomía con un 5,1\% de mortalidad y la resección hepática por metástasis con un 5,8\% de mortalidad. Cirugías complejas como la pancreatoduodenectomía o la gastrectomía total tienen un 2,9 y un 2,8\% de mortalidad respectivamente.

\section{Discusión}

El presente reporte demuestra que el trabajo de unidades como esta, permiten realizar un estudio prospectivo de los tumores malignos que se atienden en una unidad clínica. Es probable que sea la única unidad de este tipo en un hospital general del país, excluyendo los 2 hospitales dedicados exclusivamente al tratamiento del cáncer. Desconocemos la realidad de estos centros en cuanto al tipo de tumores que atienden, ya que son centros de atención integral de cualquier tipo de tumor maligno. Nuestro departamento ha sido permanentemente un centro de referencia nacional de patología digestiva alta y baja, lo que explica que 1.646 pacientes (56,6\%) del total de operados correspondan a estas localizaciones. Llaman la atención los siguientes hechos:

a) La alta tasa de cáncer de tiroides operados, correspondiendo a un tercio del total de tumores malignos, lo que significa 12 pacientes al mes. Esto se debe en parte al prestigio ganado por varios distinguidos cirujanos y por otra parte, a la dedicación exclusiva de 3 cirujanos que operan exclusivamente patología de cabeza y cuello, incluyendo tiroides y cirugía endocrinológica.

b) El sistema digestivo en el Departamento se ha subdividido en 3 secciones, cada uno de ellos dedicado a ciertos tumores: 1) Esófago-gástrico; 2) hepatobiliopancreático y 3) coloproctología. La creación y desarrollo de estos equipos desde hace varios años ha permitido, por una parte, una importante especialización en el manejo de cirugías complejas y, por otra parte, concentrar un importante número de pacientes complejos, de edad avanzada y con tumores de difícil manejo. Ha permitido también el desarrollo de la cirugía laparoscópica para el tratamiento quirúrgico de ellos, en especial para el cáncer de colon y recto, así como de esófago. También se destaca la baja mortalidad de estas cirugías pese a su alta complejidad. 
RESULTADOS DE LA UNIDAD DE ONCOLOGÍA DEL DEPARTAMENTO DE CIRUGÍA DE UN HOSPITAL ...

Como ejemplo, la mortalidad de la pancreatoduodenectomía de $2,9 \%$, la gastrectomía total de $2,8 \%$, la esofaguectomía de 5,1\% y la resección hepática por metástasis de 5,8\%; lo que demuestra la importancia de tener un hospital con alto volumen de pacientes y cirujanos con alto volumen de operaciones ${ }^{2-5}$.

c) La cirugía del cáncer mamario es muy escasa en nuestro servicio, ya que el Departamento de Ginecología ha absorbido a la mayoría de estos pacientes. Desconocemos en la realidad chilena de los hospitales públicos, quien atiende a estos pacientes, pero en clínicas privadas hay grupos dedicados sólo a esta patología, la mayoría siendo cirujanos generales.

La experiencia presentada, inédita en Chile, muestra la enorme utilidad de tener una unidad especializada inserta en los grandes servicios de cirugía del país, que permite realizar un completo estudio prospectivo y un seguimiento muy acabado, que permite tipificar las lesiones, comprobar la utilidad de los procedimientos quirúrgicos y evaluar la real sobrevida a 5 años de cada uno de los tumores de acuerdo a la etapa evolutiva de cada uno de ellos.

\section{Referencias}

1. INE. Estadísticas de Mortalidad, 2008.

2. Dimick JB, Wainess RM, Couvan JA, Upchurch GR. National trends in the use and outcome of hepatic resection. J AmColl Surg. 2004;199:31-8.

3. Schrag D, Cramer LD, Badi PB, Cohen HM, Waner $\mathrm{K}$. Influence of hospital procedure volume on outcome following surgery for colon cancer. JAMA 2000;284:3028-35.

4. Deairs KM, Smith BR, Hinojosa MW, Nguyen NT. Outcomes of esophagectomy at Academic Centers: an association between volume and outcome. Amer Surg. 2008;74:939-43.

5. Eppsteiner RW, Csikesz NG, McPee JT, Tseng J. Surgeon volume impacts hospital mortality for pancreatic resection. Ann Surg. 2009;249:635-40.

6. Allareddy V, Allareddy V, Konety BR. Specificity of procedure volume and In hospital mortality association. Ann Surg. 2007;246;135-9. 